\title{
Importance of allergological evaluation and skin testing for severe cutaneous adverse reactions: a case report
}

Philip H Li * ${ }^{*}$ MRCP (UK), FHKCP, Jane CY Wong, MB, BS, MRCP (UK), CS Lau, MD, FRCP

Division of Rheumatology and Clinical Immunology, The University of Hong Kong, Queen Mary Hospital, Pokfulam, Hong Kong

Hong Kong Med J 2020;26:444-5

*Corresponding author: liphilip@hku.hk

https://doi.org/10.12809/hkmj198263

\section{Case report}

This is the first case of acute generalised exanthematous pustulosis (AGEP) due to amoxicillin reported in Hong Kong, confirmed by complete in vivo and in vitro allergological investigations. It is vital to highlight the importance of an appropriate and thorough drug allergy evaluation for patients with a suspected causative agent.

A 24-year-old man was admitted in January 2016 to his local hospital with knee pain. Arthrocentesis was performed and empirical intravenous amoxicillin-clavulanate prescribed for suspected septic arthritis. He was also prescribed Hartmann's solution, paracetamol, tramadol, chlorpheniramine, metoclopramide, and zopiclone during his in-patient stay. A few hours later he developed fever and generalised pustulosis. There was no mucosal involvement or skin necrosis. Culture of the pustules was negative and he declined skin biopsy. However, his fever persisted and pustulosis began to worsen despite continuation of amoxicillin-clavulanate. After almost 1 week, the patient was discharged against medical advice and no other investigations were ordered. His fever subsided and rash improved without treatment. He was referred to our division 2 weeks after discharge for persistent knee pain. Upon examination, there were residual pustules with desquamation and plaques over the trunk and limbs. A diagnosis of AGEP likely to amoxicillin and/or clavulanate was suspected, agreed on review by our dermatologist. However, other possible culprits could not be excluded as he was prescribed multiple medications at the time.

He was reviewed 2 months later after improvement of his skin condition. He consented to patch testing (PT) and intradermal testing (IDT) on his back. The PT was performed using a Finn Chamber (SmartPractice, Phoenix [AZ], United States) with amoxicillin at $10 \%(2 \mathrm{mg} / \mathrm{mL})$ and $1 \%$ $(0.2 \mathrm{mg} / \mathrm{mL})$ dilutions in water. The IDT was performed with amoxicillin $20 \mathrm{mg} / \mathrm{mL}$. Immediate PT and IDT readings were negative after 20 minutes. A delayed IDT reading at 48 hours was positive with pustule formation (Fig 1). The patient declined skin biopsy and culture of the pustule was negative. The

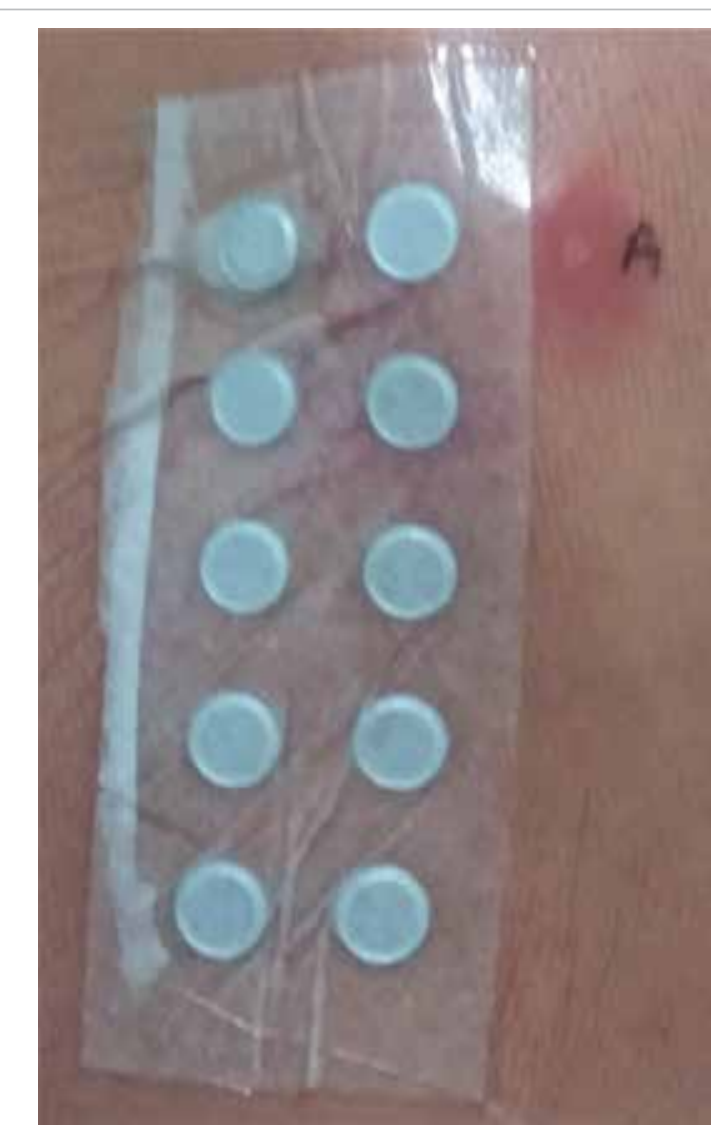

FIG I. Positive delayed intradermal test to amoxicillin $(20 \mathrm{mg} / \mathrm{mL}$ ) and with pustule formation (with uncovered patch test adjacent)

PT was strongly positive $(++)$ at D2 and D4 with a crescendo effect for amoxicillin at both $10 \%$ and $1 \%$ dilutions (Fig 2). Lymphocyte transformation test showed consistent findings, with strong positive results for amoxicillin and amoxicillin-clavulanate. The patient was advised to avoid penicillins prior to further allergological testing. He was reassured that other concomitant medications were safe. He tolerated paracetamol, tramadol, chlorpheniramine, and metoclopramide thereafter on separate occasions with no adverse effects. 


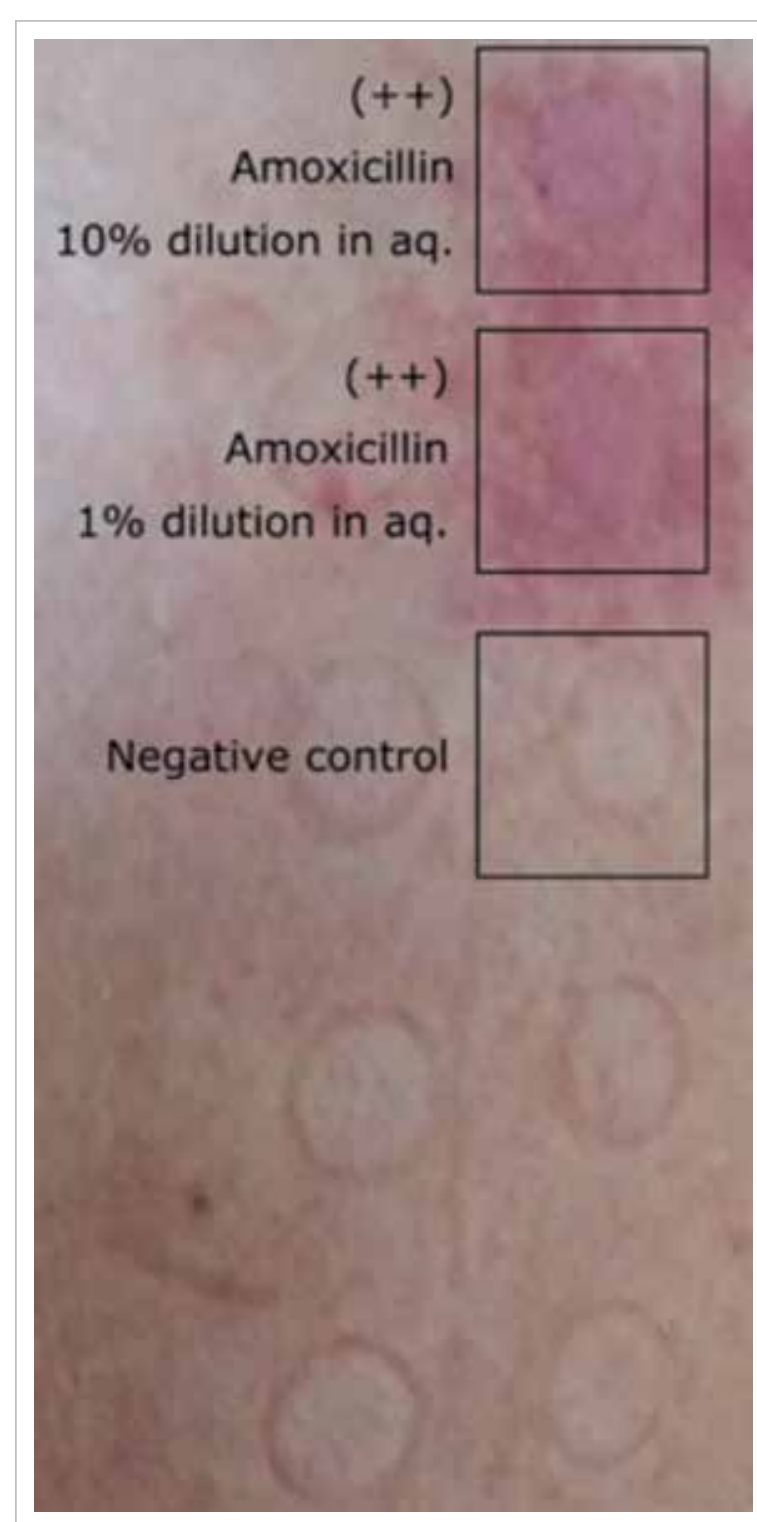

FIG 2. Positive $(++)$ patch test to amoxicillin ( $10 \%$ and I\% dilutions in aq.) on D4 differential diagnosis. Once a diagnosis of SCAR is suspected, possible causative medications should be immediately withheld. After acute management, allergological evaluation is required to identify the causative drug and prevent unnecessary avoidance of other (often multiple) medications or inadvertent re-exposure (to misidentified culprits) in future. As a type IV hypersensitivity reaction, PT and/or IDT can confirm suspected drugs as the cause of AGEP and other SCARs. ${ }^{2}$ A lymphocyte transformation test may be useful but is generally available only in research institutes. ${ }^{3}$ Lymphocyte transformation testing is highly specific, with near $100 \%$ specificity for beta-lactams. ${ }^{4}$ Physicians are reminded of the importance of comprehensive allergological evaluation to confirm suspected aetiologies in cases of SCAR.

\section{Author contributions}

Concept or design: PH Li.

Acquisition of data: PH Li.

Analysis or interpretation of data: All authors.

Drafting of the manuscript: All authors.

Critical revision of the manuscript for important intellectual content: All authors.

All authors had full access to the data, contributed to the study, approved the final version for publication, and take responsibility for its accuracy and integrity.

\section{Conflicts of interest}

All authors have disclosed no conflicts of interest.

\section{Acknowledgement}

The authors would like to thank Ms Mei-shan Lui (RN) for her aid with patch testing and outstanding service to patient care.

\section{Funding/support}

This case report received no specific grant from any funding agency in the public, commercial, or not-for-profit sectors.

\section{Ethics approval}

The patient consented to this publication.

\section{Discussion}

Acute generalised exanthematous pustulosis is a severe cutaneous adverse reaction (SCAR) that manifests with generalised sterile pustules, often mistaken for infection with subsequent inappropriate treatment. Symptoms classically appear within hours, especially in antibiotic-triggered reactions. ${ }^{1}$ Differential diagnoses of pustular skin eruptions should be considered including pustular psoriasis, hypersensitivity syndrome reaction with pustulation, and subcorneal pustular dermatosis. However, given the clear chronological administration timeline of a suspected drug, AGEP should remain the prime

\section{References}

1. Alvarado SA, Muñoz-Mendoza D, Bahna SL. High-risk drug rashes. Ann Allergy Asthma Immunol 2018;121:55260.

2. Barbaud A, Gonçalo M, Bruynzeel D, Bircher A, European Society of Contact Dermatitis. Guidelines for performing skin tests with drugs in the investigation of cutaneous adverse drug reactions. Contact Dermatitis 2001;45:321-8.

3. Mayorga C, Celik G, Rouzaire P, et al. In vitro tests for drug hypersensitivity reactions: an ENDA/EAACI Drug Allergy Interest Group position paper. Allergy 2016;71:1103-34.

4. Doña I, Torres MJ, Montañez MI, Fernández TD. In vitro diagnostic testing for antibiotic allergy. Allergy Asthma Immunol Res 2017;9:288-98. 